\title{
Experimental and theoretical Determination of Low electron Energy Loss spectra of $\mathrm{Ag}$ and $\mathrm{Ru}$
}

\author{
C. Hebert ${ }^{a}$, W.-D. Schöne ${ }^{\text {b }}$ D. S. Su ${ }^{c}$ \\ anstitut für Festkörperphysik, Technische Universität Wien, A-1040 Wien \\ ${ }^{b}$ Department of Physical Chemistry, Fritz-Haber- Institut of the Max-Planck-Society, \\ Faradayweg 4-6, D-14195 Berlin \\ ${ }^{\mathrm{C}}$ Department of Inorganic Chemistry, Fritz-Haber- Institut of the Max-Planck-Society, \\ Faradayweg 4-6, D-14195 Berlin
}

\begin{abstract}
We show the experimental and calculated q-dependent low energy loss electron energy loss spectrum of $\mathrm{Ru}$ and $\mathrm{Ag}$. The spectra were calculated within the timedependent density-functional theory including local-field effects. For Ag, the momentum transfer was parallel to the (110) direction. For Ru the 3 main directions (010), (110) and (001) were investigated. The agreement between theory and experiment is very good for $\mathrm{Ag}$ and momentum transfers parallel to the (001) direction of $\mathrm{Ru}$. For momentum transfers parallel to the in-plane directions (110) and (010) the agreement for Ru is not satisfactory, which could be attributed to relativistic effects or strong localization of the $4 d$ states of $\mathrm{Ru}$.
\end{abstract}

Key words: Electron Energy Loss spectroscopy, linear response 